\title{
D. Franzoni, La prosa sovietica nel contesto socio-culturale dell'epoca brežneviana, FUP, Firenze 2020, pp. 170.
}

Per chi ha l'età per ricordare l'interesse smisurato di cui era oggetto negli anni Ottanta (non solo in Unione Sovietica) e la repentina caduta di questo interesse nel decennio successivo, trovare oggi Čingiz Ajtmatov tra gli eroi di un saggio che punta a riscrivere una pagina di storia della letteratura (della letteratura, e non del costume) non può che costituire una sorpresa (una sorpresa dal sapore dolceamaro, ma non è questo il punto).

La felice idea di fondo del libro di Franzoni è quella di riprendere, rivalutare, nel periodo della cosiddetta 'stagnazione' brežneviana, periodo di cui oggi studiamo soprattutto la letteratura clandestina, underground (o andegraund), la letteratura che chiama "consentita" - una letteratura, cioè, che si pubblica sulla stampa sovietica, senza per questo potere, o dovere, essere definita letteratura 'ufficiale' o essere considerata espressione diretta delle intenzioni del regime: gli autori scelti come oggetto principale di indagine - i capitoli monografici sono dedicati a Rasputin, Šukšin, appunto Ajtmatov e Trifonov - sono quasi tutti autori che camminano in equilibrio più o meno precario sullo steccato dell'ammissibile. Autori, senza dubbio, degni di un interesse attento, che oggi sono scarsamente trattati dalla critica letteraria, e mai insieme. Un' indagine dei rapporti tra questa letteratura e quella intenzionalmente sommersa, rapporti probabilmente più intensi e complessi di quanto non si tenda a dare oggi per scontato, aiuterebbe a precisare meglio i contorni della mappa. Ma questa non è una mancanza da imputare all'autore (che ha tutto il diritto di porre dei limiti al suo campo di indagine) quanto un suggerimento per una ricerca futura; non avrebbe senso valutare un lavoro di questo tipo per quello che non contiene: ogni lettore con un minimo di competenza potrebbe indicare, a seconda delle sue preferenze, questo o quell'autore che si sarebbe potuto prendere in considerazione. Oggetto del giudizio su un libro del genere dev'essere quello che nel libro c'è.

E quello che c'è in questo libro è, nonostante l'autore si sforzi di negarlo, il tentativo di presentare, per il suo campo limitato, un canone (i paragrafi introduttivi ai capitoli monografici rendono conto di un contesto più ampio, sono presi in considerazione, oltre agli autori già citati, tra gli altri Astaf 'ev, Iskander, Makanin). Un tentativo del genere, per di più rispetto ad un'epoca storica ancora viva nella memoria di alcuni, è destinato, gioco forza, a suscitare discussioni. Chi scrive, per esempio, resta convinto che - all'interno del tentativo, estremamente interessante, di tracciare un quadro di una letteratura 'sovietica', letta in russo ma prodotta originariamente anche in altre lingue nelle repubbliche periferiche - la scelta di privilegiare Ajtmatov rispetto, per esempio, a Fazil' Iskander non sia particolarmente felice (è questo un caso in cui, forse, la scelta di escludere dal quadro tut- 
to quanto non sia stato pubblicato e dibattuto dalla stampa sovietica mostra qualche limite). Così come quella di trattare Rasputin e, ancora di più, Šukšin lasciando la questione della lingua come in qualche modo secondaria e accessoria rispetto agli intenti di analisi sociale o morale.

Si potrebbe continuare. È un libro, insomma, destinato a far discutere: evento piuttosto raro nella slavistica italiana. Tanto che il capitolo più interessante e coraggioso (più interessante e più coraggioso di quelli monografici che, per quanto ben documentati e ben condotti, raramente stravolgono il ritratto già noto degli scrittori in oggetto), è il capitolo che tira le somme. L'analisi complessiva della letteratura 'consentita' mette qui in evidenza sia i compromessi cui gli autori sono costretti per accedere alla possibilità di pubblicare, sia le aperture che si distaccano dai canoni realisti socialisti in modo quasi sorprendente, dimostrando una vitalità della letteratura sovietica degli anni Settanta raramente riconosciuta nell'immagine contemporanea, e arrivando a proporre di riconsiderare l'applicabilità del termine 'stagnazione' al campo letterario.

Il libro di Franzoni, insomma, riporta all'attenzione un periodo, e una serie di autori, oggi quasi dimenticati dalla critica, che meritano invece di essere ripresi in considerazione in prospettiva storica; lo fa avanzando con coraggio affermazioni a volte discutibili. Ha dunque, vale ripeterlo, il pregio raro di essere in grado di provocare dibattito: difficile immaginare un merito maggiore per un testo di storia letteraria.

Duccio Colombo 